\title{
Alpha heating in ITER L-mode and H-mode plasmas
}

\author{
R.V. Budny \\ Princeton Plasma Physics Laboratory, Princeton, NJ 08543, USA \\ E-mail: budny@princeton.edu
}

Received 16 December 2010, accepted for publication 1 November 2011

Published 28 November 2011

Online at stacks.iop.org/NF/52/013001

\begin{abstract}
Predictions of alpha heating in ITER L-mode and H-mode DT plasmas are generated using the PTRANSP code. The baseline toroidal field $(5.3 \mathrm{~T})$, plasma current ramped to $15 \mathrm{MA}$ and a flat electron density profile ramped to Greenwald fraction 0.85 are assumed. Various combinations of external heating by negative ion neutral beam injection, ion cyclotron resonance and electron cyclotron resonance are assumed to start half-way up the density ramp with the full power planned $\left(P_{\text {ext }}=73 \mathrm{MW}\right) .50 \mathrm{~s}$ later the power is reduced to $50 \mathrm{MW}$ to increase $Q_{\mathrm{DT}}$, and to prevent excessive heat flow to the divertor and walls as the alpha heating increases. The time evolution of plasma temperatures and bulk toroidal rotation $v_{\phi}$ are predicted assuming GLF23 and boundary parameters. Conservatively low temperatures $(\simeq 0.6 \mathrm{keV})$ and $v_{\phi} \simeq 400 \mathrm{rad} \mathrm{s}^{-1}$ at the boundary $(r / a \simeq 0.85)$ are assumed.

Alternative options are used to predict $v_{\phi}$ and the flow-shearing rates induced by the neutral beam torques in order to assess effects of uncertainties. Option 1 assumes the momentum transport coefficient $\chi_{\phi}$ is half the energy transport coefficient $\chi_{i}$ predicted consistently with the GLF23-predicted temperatures. With this assumption flow shearing does not have large effects on the energy transport, plasma temperatures and alpha heating. Option 2 uses GLF23 to predict $v_{\phi}$ directly. Higher flow-shearing rates and alpha heating powers are predicted for heating mixes with neutral beam heating. If the $\mathrm{L} \rightarrow \mathrm{H}$ power threshold is twice the ITPA fit then the heating mixes with the highest neutral beam power (and the most alpha heating) transition to H-mode during the density ramp. Other heating mixes remain in L-mode.

Predictions of H-mode temperatures and alpha heating depend sensitively on the assumed pedestal pressures. A scan in pedestal pressures is presented using the more pessimistic option 1. A linear increase in alpha heating with pedestal temperature and pressure is predicted.
\end{abstract}

\section{Introduction}

One of the goals of ITER [1] experiments will be to produce plasmas with a fusion gain $Q_{\mathrm{DT}}$ (ratio of the DT fusion and external heating powers, $\left.P_{\mathrm{DT}} / P_{\mathrm{ext}}\right) \geqslant 10$. Enhanced confinement regimes such as the $\mathrm{H}$-mode appear necessary to achieve this, but the physics basis for the $\mathrm{L} \rightarrow \mathrm{H}$ transition as well as confinement enhancement remain unclear, and extrapolations of database scaling might not be reliable for predicting the heating power needed for the transition. Thus having the capability of large amounts of external heating power should increase the probability of success. The planned heating and current drive systems for ITER are negative ion neutral beam injection (NB), ion cyclotron resonance frequency (IC) and electron cyclotron resonance frequency (EC) with maximum total power of $73 \mathrm{MW}$. Lower-hybrid current drive is planned as a possible later upgrade.

The PTRANSP code [2-4] is used to generate timedependent integrated predictions. Time dependence is modelled to include plasma formation, termination and transients such as magnetic field diffusion and sawtooth effects. PTRANSP ITER predictions are being used for various applications such as designing diagnostics [5-7], and for use in theory studies of Alfvén eigenmodes [8].

Here PTRANSP is used to predict conditions in L-mode and $\mathrm{H}$-mode plasmas. Several expressions for the H-mode power threshold are coded into PTRANSP. The one used here is an ITPA database fit [9]:

$$
\begin{aligned}
& P_{\mathrm{ITPA}}=2.15 \kappa^{ \pm 0.107} n_{\mathrm{e} 20}^{0.782 \pm 0.037} B_{\text {tesla }}^{0.772 \pm 0.031} a_{\mathrm{m}}^{0.975 \pm 0.08} \\
& \quad \times R_{\mathrm{m}}^{0.999 \pm 0.01} 2.0 / M_{\mathrm{AMU}}(M W) .
\end{aligned}
$$

Here $\kappa$ is the elongation of the boundary and $M$ is the average isotopic mass of the hydrogenic species. Note that this scaling decreases with decreasing electron density indicating that it might be beneficial to start NB injection early. Some experiments indicate a configuration-dependent minimum in density [10-12] not given by this scaling. However, recent JET experiments [13] with a recent divertor configuration show no minimum.

It is unclear whether and at what $n_{\mathrm{e}}$ this density minimum would occur in ITER. Here the external heating is started at 
Table 1. The six heating power mixes considered. Values for the NB-IC-EC heating powers in MW are listed for four time periods.

\begin{tabular}{lllll}
\hline Heating mix & $80-130 \mathrm{~s}$ & $130-300 \mathrm{~s}$ & $300-400 \mathrm{~s}$ & $400-500 \mathrm{~s}$ \\
\hline 2NB/IC/EC & $33-23-20$ & $33-17-0$ & $33-7-0$ & $0-0-4$ \\
2NB/EC & $33-0-40$ & $17-0-30$ & $17-0-17$ & $0-0-4$ \\
2NB/EC/IC & $33-20-20$ & $17-10-20$ & $17-0-17$ & $0-0-4$ \\
$1 \mathrm{NB} / \mathrm{IC} / \mathrm{EC}$ & $17-20-37$ & $0-10-37$ & $0-0-33$ & $0-0-4$ \\
IC/EC & $0-20-53$ & $0-10-36$ & $0-0-33$ & $0-0-4$ \\
EC & $0-0-73$ & $0-0-46$ & $0-0-33$ & $0-0-4$ \\
\hline
\end{tabular}

half the flat top density. Full-power NB cannot start too early since the beam shine-through could damage the first wall.

Plasma parameters near the boundary within the separatrix are needed for the temperature predictions. The PEDESTAL module [14] in PTRANSP can be used to predict the pedestal width and the pressure at the top of the pedestal after the H-mode transition. Since the electron density is prescribed, the pressure determines the temperatures. These boundary values can be scaled, and for the L-mode study the flat top values of both the ion and electron temperatures after the transition are assumed to be $4.4 \mathrm{keV}$. A power threshold for the back transition $P_{H \rightarrow L}$ is also modelled and the H-mode phase is terminated when $P_{\text {heat }} \equiv P_{\text {ext }}+P_{\alpha}$ decreases below a pre-set factor of $P_{\mathrm{L} \rightarrow \mathrm{H}}$. Here $P_{H \rightarrow L} / P_{\mathrm{L} \rightarrow \mathrm{H}}=75 \%$ is assumed.

The full external power $P_{\text {ext }}=73 \mathrm{MW}$ is sufficient to achieve the H-mode at half-density if $P_{\mathrm{L} \rightarrow \mathrm{H}}=P_{\text {ITPA }}$ (equation (1)). In this case no L-mode phase is expected after the external heating is started. The alpha heating $P_{\alpha}$ during the ohmically heated L-mode at half density is predicted to be relatively small $(\simeq 0.3 \mathrm{MW})$. Here, to study externally heated L-modes in ITER, expression equation (1) is multiplied by either two or four. Even with $P_{\mathrm{L} \rightarrow \mathrm{H}}=2 \times P_{\text {ITPA }}$, $P_{\text {ext }}=73 \mathrm{MW}$ would be insufficient alone to achieve the $\mathrm{H}$-mode, and additional alpha heating would be needed. Thus the power from alpha self-heating in the L-mode phase could play a crucial role in achieving the $\mathrm{H}$-mode. In the factorof-two case the additional alpha heating is predicted to be sufficient for the H-mode transition for some of the heating mixes considered.

ITER L-mode predictions are in $[4,15]$. Here a wider range of assumptions, both more and less pessimistic are used to explore a range of possibly results in ITER. Predictions of ITER H-mode $P_{\mathrm{DT}}$ with a scan in boundary temperatures $T_{\text {ped }}$ are in $[2,15]$. Here a wider scan in the corresponding normalized pedestal pressure $\beta_{\mathrm{n} \text {,ped }}$ is studied and results for a wider range of plasma parameters are given.

\section{Plasmas studied}

Six mixes of external heating are considered, and summarized in table 1, with heating power waveforms plotted in figure 1 . The first five are similar to those considered in [4]. Each NB beamline is specified to deliver up to $P_{\mathrm{NB}}=16.5 \mathrm{MW}$ of $1 \mathrm{MeV} \mathrm{D}{ }^{0}$. The baseline design includes two beamlines. The last mix with only EC is not being planned for ITER, but is considered here as an alternative in case the NB and IC developments are unsuccessful. Comparisons of the merits of four heating mixes are discussed in [16]. That paper concluded that plasmas with IC heating would achieve higher $Q_{\mathrm{DT}}$ than those with NB heating. Here mixes having more IC heating have lower $Q_{\mathrm{DT}}$ than those with more NB heating.

The NUBEAM module [17] in PTRANSP is used to simulate the NB heating, torquing, current drive and alpha heating. Below-axis beam NB steering is assumed, with the centre of the beams displaced $25 \mathrm{~cm}$ below the vessel midplane at the 'turning point' of minimum major radius $(R=5.295 \mathrm{~m})$ as described in [2]. The IC is assumed to use $\mathrm{He}^{3}$ minority heating at $52.5 \mathrm{MHz}$ for central heating. The $\mathrm{He}^{3}$ concentration is assumed to be $2 \% n_{\mathrm{e}}$. The baseline design specifies capability of $20 \mathrm{MW}$ injected power. The TORIC full-wave code [18], coupled into PTRANSP is used to simulate the IC heating. TORIC simulations have been benchmarked with other full-wave solvers [19] using PTRANSP predicted ITER plasmas. The planned EC heating and current drive frequency is $170 \mathrm{GHz}$, launched in O-mode. The baseline design specifies capability of $20 \mathrm{MW}$ injected power. Three midplane launchers and two upper launchers (intended for neoclassical tearing mode (NTM) suppression) are planned. The assumed launcher positions and angles are those in table 2 of [4]. The TORAY-GA code [20-22] is used to model the heating and current drive.

Standard assumptions are used for the toroidal magnetic field $B_{\text {tor }}=5.3 \mathrm{~T}$, plasma current $I_{\mathrm{p}}$ ramped to $15 \mathrm{MA}$ and Greenwald fraction $\bar{n}_{\mathrm{e}} / \bar{n}_{\mathrm{GW}}$ (with $\bar{n}_{\mathrm{e}}$ the line-average and $\left.\bar{n}_{\mathrm{GW}} \equiv I_{\mathrm{p}} /\left(\pi a^{2}\right) \times 10^{20} \mathrm{~m}^{-3}\right)$ ramped to 0.85 . The assumed ramp-up of the central electron density and the computed ion densities are shown in figure $2(a)$.

\section{PTRANSP}

In PTRANSP the sum of the thermal deuterium and tritium density profiles is computed from the assumed $n_{\mathrm{e}}$ and the selfconsistently computed fast-ion and impurity densities. This is not sufficient to specify the separate $n_{\mathrm{D}}$ and $n_{\mathrm{T}}$ profiles, but they can be computed using one of a variety of user-controlled mixing models. The model used for this study specifies relative diffusivities and pinches for the $\mathrm{D}$ and $\mathrm{T}$. This choice has been used to analyse DT experiments in TFTR and JET predicting the DT neutron emission in approximate agreement with measurements from neutron colluminator data [23]. The prediction for ITER is that the $n_{\mathrm{D}}$ and $n_{\mathrm{T}}$ are nearly equal. The plasma current and various components for one of the heating mixes are shown in figure $2(b)$.

Previous PTRANSP papers [2-4] use physics-based models to predict profiles of the temperatures $T_{\mathrm{e}}$ and $T_{\mathrm{i}}$, and in some cases, the toroidal rotation $v_{\text {tor }}$. The GLF23 model [24] is used here. An improved model TGLF [25] achieves more accurate predictions of temperatures measured in DIIID, JET and TFTR. That model requires parallel processing for much longer calculations per call than GLF2, and it has not yet been coupled into comprehensive self-consistent transport codes. GLF23 does achieve approximate agreement predicting temperatures and $v_{\phi}$. Examples of predictions are in [26].

There are various options and adjustable parameters for running GLF23. The most extensively tested options use measured $v_{\phi}$ rates, and radial force balance to compute flowshearing rates and use these rates with the GLF23 predicted 

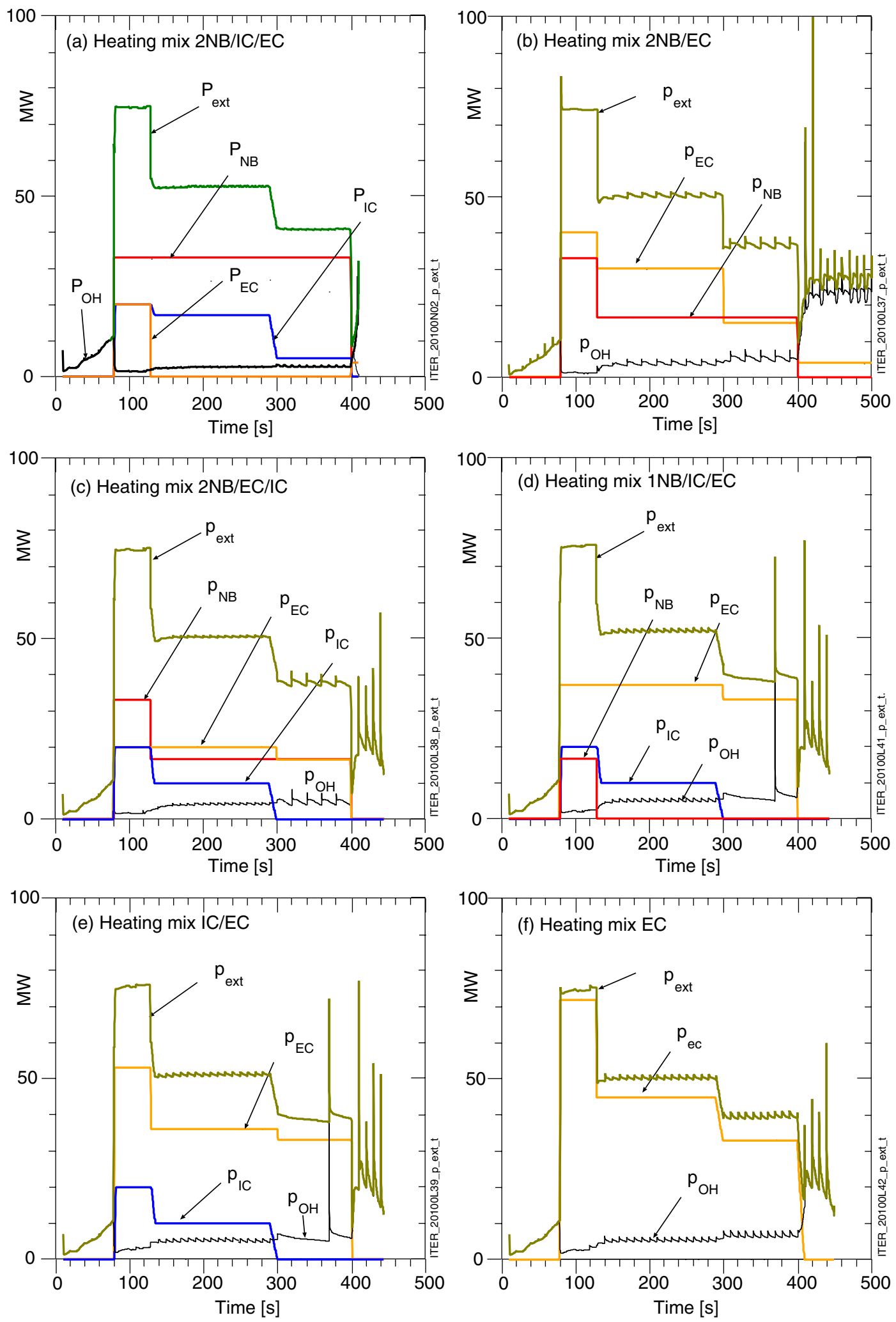

Figure 1. Powers assumed for the heating mixes. The ohmic power for each mix varies as the resistivity varies according to the choice of the option and $P_{\mathrm{L} \rightarrow \mathrm{H}}$. The runs end with a thermal collapse when the heating power becomes too low.

ion and electron transport coefficients $\chi_{\mathrm{i}}$ and $\chi_{\mathrm{e}}$ to predict ion and electron temperatures. Alternatively $v_{\phi}$ can be predicted and used to compute flow-shearing rates which can be used to predict self-consistently ion and electron temperatures. Both options are considered here. For option $1 v_{\phi}$ is predicted assuming that the ratio of the momentum transport $\chi_{\phi}$ to the GLF23-predicted $\chi_{i}$ is 0.5 , which is roughly midway between values measured in tokamaks with co-plasma-current 
Table 2. L-mode values for $P_{\alpha}$ and the time span of the H-mode phase. For options 1 and 2 assuming $P_{\mathrm{L} \rightarrow \mathrm{H}} / P_{\mathrm{ITPA}}=4$, the maximum $P_{\alpha}$ occurs at $130 \mathrm{~s}$, just before $P_{\text {ext }}$ is lowered. No $\mathrm{H}$-mode is achieved. For option 2 assuming $P_{\mathrm{L} \rightarrow \mathrm{H}} / P_{\mathrm{ITPA}}=2$, three of the mixes transition to $\mathrm{H}$-mode and the maximum L-mode values for $P_{\alpha}$ occur just before the transition.

\begin{tabular}{llllll}
\hline Heating mix & \multicolumn{2}{l}{ Option 1} & \multicolumn{4}{c}{ Option 2} \\
\cline { 2 - 3 } \cline { 5 - 6 }$P_{\mathrm{L} \rightarrow \mathrm{H}} / P_{\mathrm{ITPA}}$ & 4 & & 4 & 2 & 2 \\
& $P_{\alpha}(\mathrm{MW})$ & & $P_{\alpha}(\mathrm{MW})$ & H-mode span $(\mathrm{s})$ & $P_{\alpha}(\mathrm{MW})$ \\
\hline 2NB/IC/EC & 16.0 & 60 & $100.1-400.0$ & 22 \\
2NB/EC & 13.3 & 62 & $108.2-400.0$ & 41 \\
2NB/EC/IC & 16.6 & & 34 & $110.1-311.0$ & 41 \\
1NB/IC/EC & 14.2 & 28 & none & 28 \\
IC/EC & 12.5 & 13 & none & 13 \\
EC & 8.4 & 8 & none & 8 \\
\hline
\end{tabular}
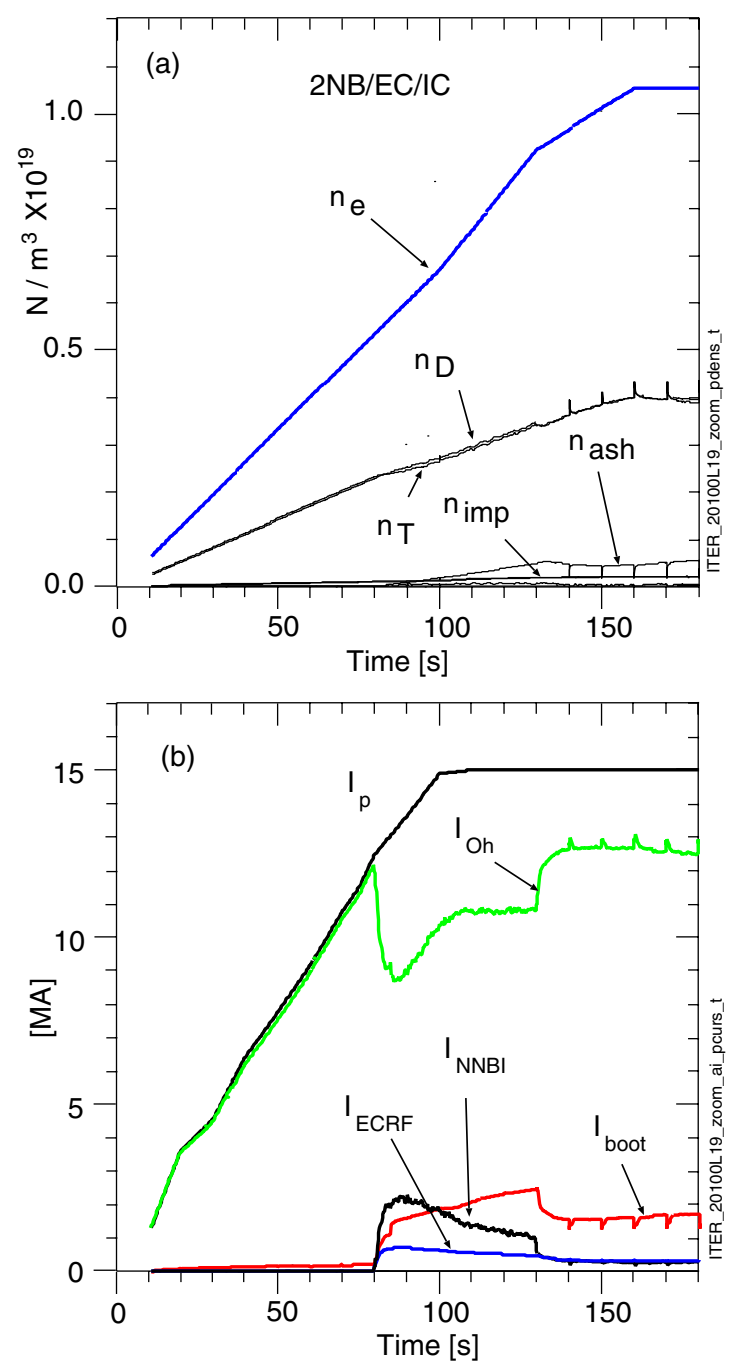

Figure 2. (a) Assumed central electron density ramped up to $f_{\text {Greenwald }}=0.85$, and computed central ion densities; $(b)$ current ramped up to $15 \mathrm{MA}$.

neutral beam injection. The $\chi_{i}$ profile is calculated in GLF23 consistently with the flow shear taken into account.

For option 2, $v_{\phi}$ is predicted by GLF23, so the predictions of temperatures and $v_{\phi}$ are consistent. These predictions have been tested [26], but not as extensively as the predictions using option 1. Predictions using option 2 suggest significant improvement of $Q_{\mathrm{DT}}$ in ITER H-mode plasmas [27]. The momentum predictions of GLF23 do not have as rigorous a physics basis [28] as the temperature predictions do. Nevertheless, both options are used here to assess effects of uncertainties. Option 2 gives much more optimistic predictions for ITER L-mode plasmas with the heating mixes using NB.

The flow-shearing rate in GLF23 is a constant times the gradient of the $E \times B$ velocity. The profile of the electric field $E$ is computed balancing the pressure gradient and the Lorentz force computed using the toroidal and poloidal magnetic fields, the toroidal and poloidal velocities. The latter are computed using NCLASS [29]. The value of 1.35 for the constant is often used in comparisons with experiments. Here it is assumed conservatively to be unity. The toroidal rotation is computed using GLF23 and the torques predicted for the NB. The boundary values for the temperatures and $v_{\phi}$ are taken at the flux surface with $x$ (defined as the square-root of the normalized toroidal flux) at 0.85 . The profile of $\chi_{i}$ is assumed to be neoclassical in the core since GLF23 is not valid there.

The impurities are assumed to be the $\mathrm{He}^{3} \mathrm{IC}$ minority, $\mathrm{Be}$, $\mathrm{Ar}$ and $\mathrm{He}$ ash. The Be and Ar density profiles are assumed to be $2 \%$ and $0.12 \%$ of the electron density $n_{\mathrm{e}}$. Ash transport and recycling are also modelled following [2]. The ash recycling coefficient is assumed to be 0.7 , and the ash diffusivity is assumed to be $1 \mathrm{~m}^{2} \mathrm{~s}^{-1}$ with an inward pinch of $1 \mathrm{~m} \mathrm{~s}^{-1}$. These values are consistent with profiles of the transport measured in TFTR DT L-mode plasmas and in JT-60U D plasmas.

The poloidal field diffusion and ohmic heating are calculated using neoclassical resistivity predicted by NCLASS, and the calculated $T_{\mathrm{e}}$ and $Z_{\text {eff }}$ profiles. Radiation losses and effects of charge-exchange losses due to collisions with recycled neutrals are included. The total radiation loss is predicted to be about $30 \mathrm{MW}$. The total net charge-exchange loss is predicted to be confined to the edge region beyond $x=0.9$ and to be relatively small. The atomic cross sections used are those from the ADAS project [30,31].

Sawtooth mixing is expected to have significant effects on the fast alpha particles. Sawteeth effects are modelled using a Kadomtsev-like mixing. At the time of each requested sawtooth break PTRANSP checks the $q$ profile for $q=1$ flux surfaces. If there is only one, the current, densities and temperatures are mixed conserving helical flux at strengths that are user-controlled. Here full mixing is assumed. The Porcelli model for calculating conditions for sawtooth break times installed in PTRANSP [3] can be used to predict sawtooth crash times, but the implementation has not been tested thoroughly. Here a constant sawtooth period of $10 \mathrm{~s}$ is assumed.

The electron density here is assumed fixed and unmixed by sawteeth. Since the $\mathrm{He}^{3}, \mathrm{Be}$ and Ar densities are assumed to be proportional to $n_{\mathrm{e}}$ they also remain unmixed. The beam, alpha and thermal deuterium, tritium and ash ions mix maintaining local charge neutrality. The thermal ash is mixed outwards forming a brief $\left(\simeq 1 \mathrm{~s}\right.$ ) hollow $n_{\text {ash }}$ profile. The core thermal deuterium and tritium ions increase transiently to compensate. This mixing causes a transient burst in $P_{\mathrm{DT}}$. Details are given in section 3.2 of [2]. There are lots of uncertainties about the effects of sawtooth mixing. The sawtooth mixing of fast alpha particles used here was tested with measurements in TFTR [32]. 

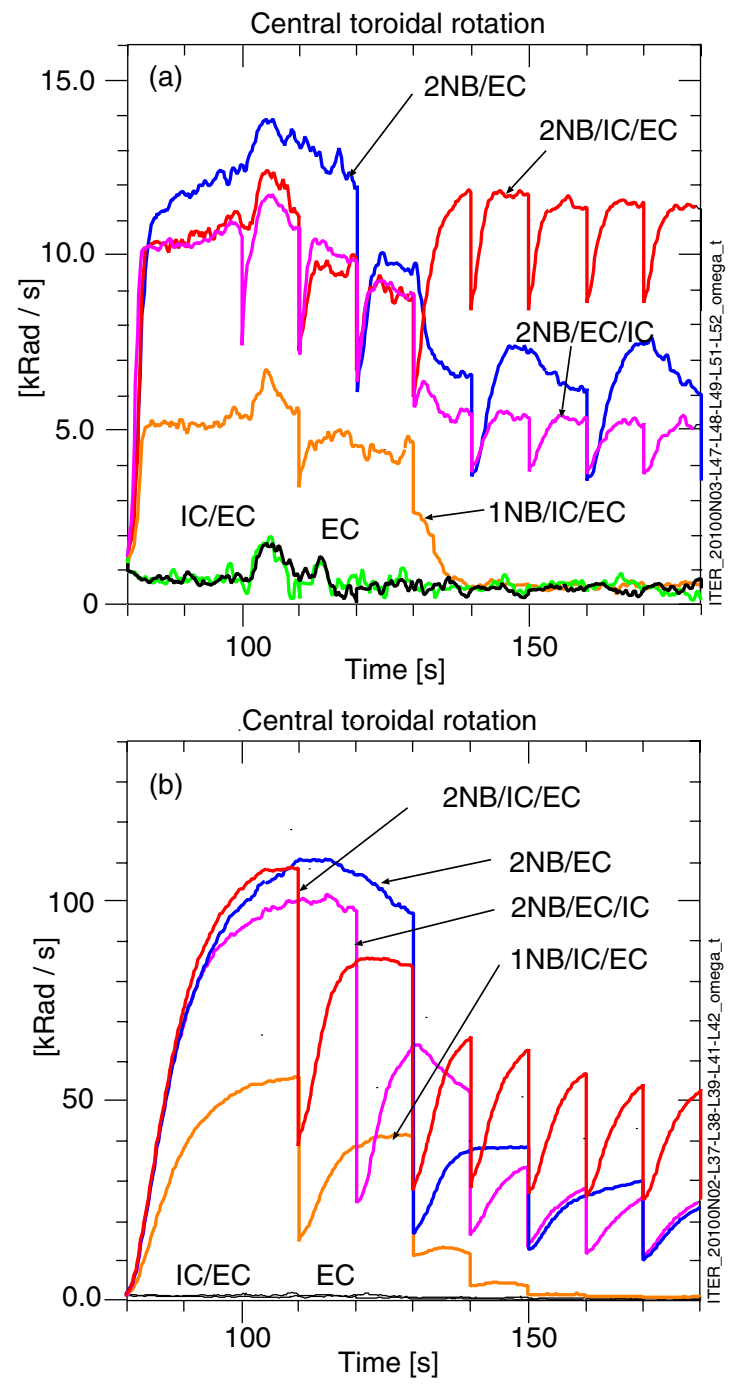

Figure 3. Central toroidal rotation for different heating mixes predicted using $(a)$ option $1\left(\chi_{\phi}=0.5 \chi_{\mathrm{i}}\right) ;(b)$ option 2 (GLF23 rotation) and $4 \times P_{\mathrm{L} \rightarrow \mathrm{H}}$. The peak rotation is much higher using option 2, but is comparable to rates measured in some L-mode beam-heated plasmas.

\section{Results for the L-mode}

Conservatively low $v_{\phi} \simeq 400 \mathrm{rad} \mathrm{s}^{-1}$ at the boundary $(r / a=$ $0.85)$ is assumed. Evolutions of the predicted central $v_{\phi}$ using option 1 and assuming high $P_{\mathrm{L} \rightarrow \mathrm{H}}$ relative to $P_{\text {heat }}$ (and $P_{\text {ITPA }}$ ) are shown in figure $3(a)$. These rotation rates are modest compared with the rates often measured in tokamaks with neutral beam injection delivering net torque.

The temperatures at the boundary for the L-mode are also assumed to be conservatively low, as shown in figure 4(a). GLF23-predicted temperature profiles are shown in figure 5. The total thermal ion and electron heating are plotted in figure 6(a). These predictions remain below the assumed $P_{\mathrm{L} \rightarrow \mathrm{H}}$. Differences of flows predicted for the different heating mixes are relatively small, indicating that flow shear suppression effects are not large.

Plots of the alpha heating $P_{\alpha}$ are shown in figure 7(a). The peak $P_{\alpha}$ is $\simeq 17 \mathrm{MW}$ (for heating mixes using two beamlines) and decreases $50 \mathrm{~s}$ later (at $130 \mathrm{~s}$ ) when $P_{\text {ext }}$ is reduced. The
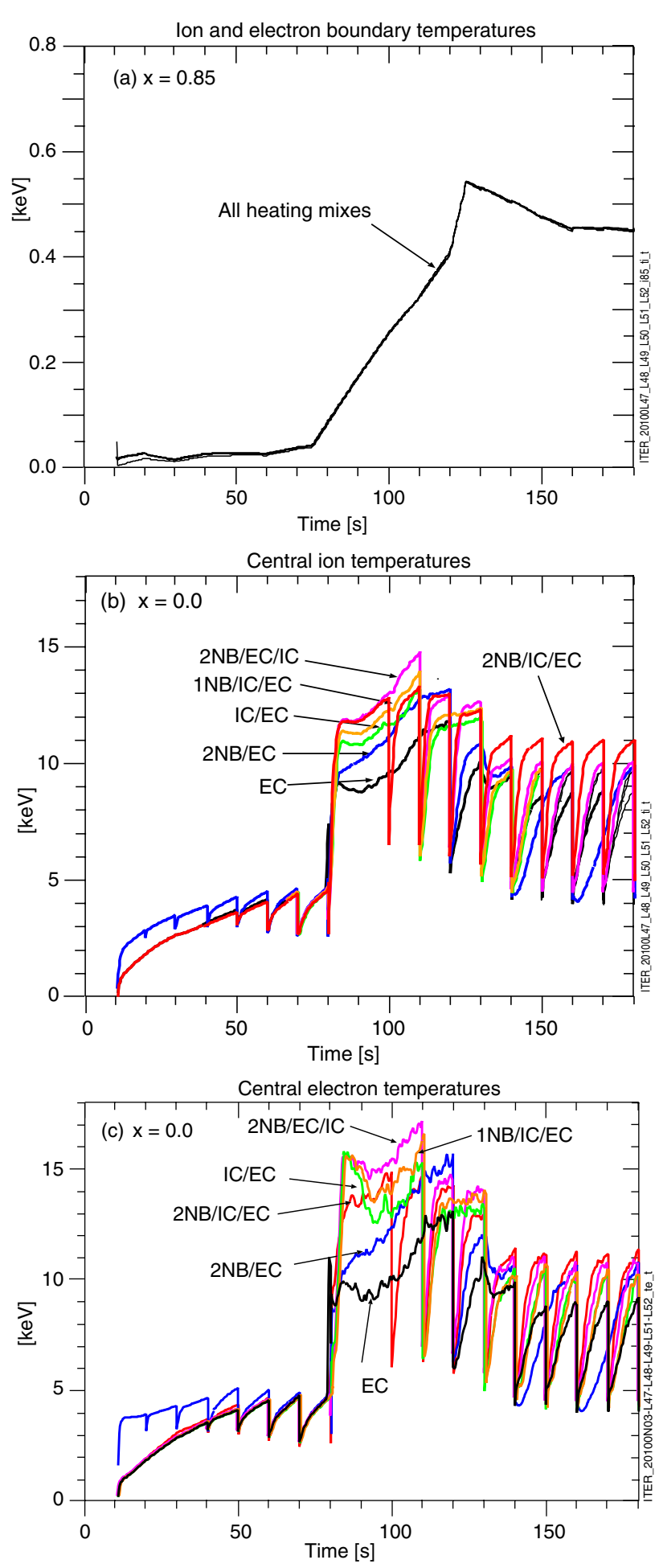

Figure 4. Predictions with different heating mixes using option 1 and high $P_{\mathrm{L} \rightarrow \mathrm{H}}$ for $(a)$ ion and electron temperatures assumed at the boundary; $(b)$ ion and $(c)$ electron temperatures computed at the magnetic axis.

peak $P_{\mathrm{DT}}$ is $\simeq 65 \mathrm{MW}$ with $P_{\mathrm{ext}}=73 \mathrm{MW}$. The values of $P_{\alpha}$ at the end of the high $P_{\text {ext }}$ phase are listed in table 2. $Q_{\mathrm{DT}}(t)$ shown in figure $8(a)$ are below unity except during sawtoothinduced transients when $P_{\text {ext }}=73 \mathrm{MW}$, or is reduced to 37 MW. The relatively large off-axis beam-driven current and the sawtooth mixing of the beam ions conspire to make the 

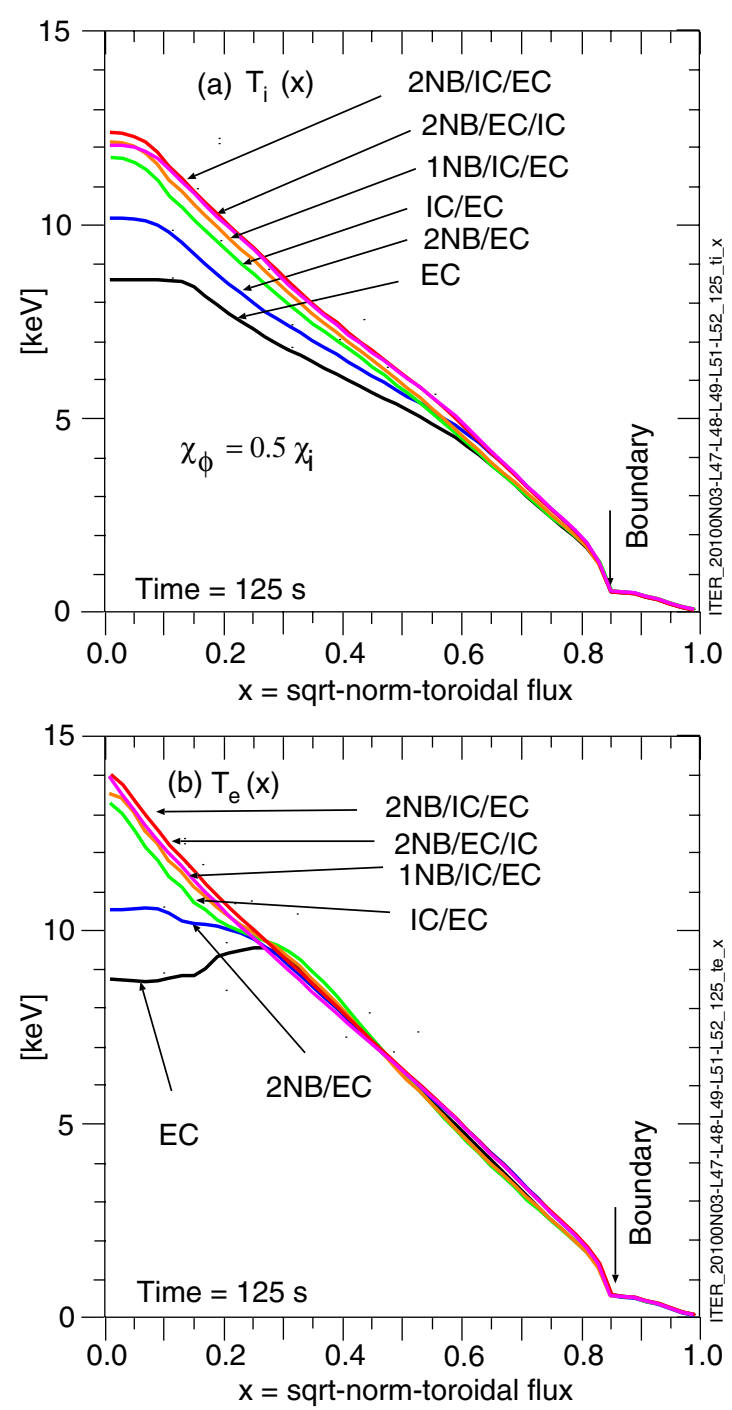

Figure 5. ( $a$ ) Ion, and $(b)$ electron temperature profiles predicted using option 1 near the time when they are largest. Boundary values with local $\beta_{\mathrm{n}}=0.1$ are specified.

$q$ profile non-monotonic near the magnetic axis with a very large mixing radius $(x \simeq 0.7)$. The mixing of the ion densities over this large volume is predicted to cause a large increase in $P_{\mathrm{DT}}$ for about $1 \mathrm{~s}$. For some heating mixes cycles of alternate large and small crashes are predicted. Also the beam current drive can prevent some of the requested sawtooth trigger times from mixing. There is considerable uncertainty about sawtooth mixing effects in ITER.

With option 2 much higher $v_{\phi}$ rates and fusion yields are predicted. First consider the case where $P_{\mathrm{L} \rightarrow \mathrm{H}}$ is higher than $P_{\text {heat }} \equiv P_{\text {ext }}+P_{\alpha}$ for all the heating mixes. $P_{\mathrm{L} \rightarrow \mathrm{H}}=$ $4 \times P_{\text {ITPA }}$ is sufficiently high. Central rotation rates are shown in figure $3(b)$. The boundary temperatures are the same as those used for option 1 and shown in figure 4(a). The peak temperatures are shown in figure 9. The ion temperatures are relatively high for the heating mixes with high NB power (and relatively large $v_{\phi}$ rates). The peak values occur as the density is ramping up. Similar phenomena are observed in the supershot regime in TFTR and the hot ion H-mode in JET. This results from the stiffness of GLF23 and the
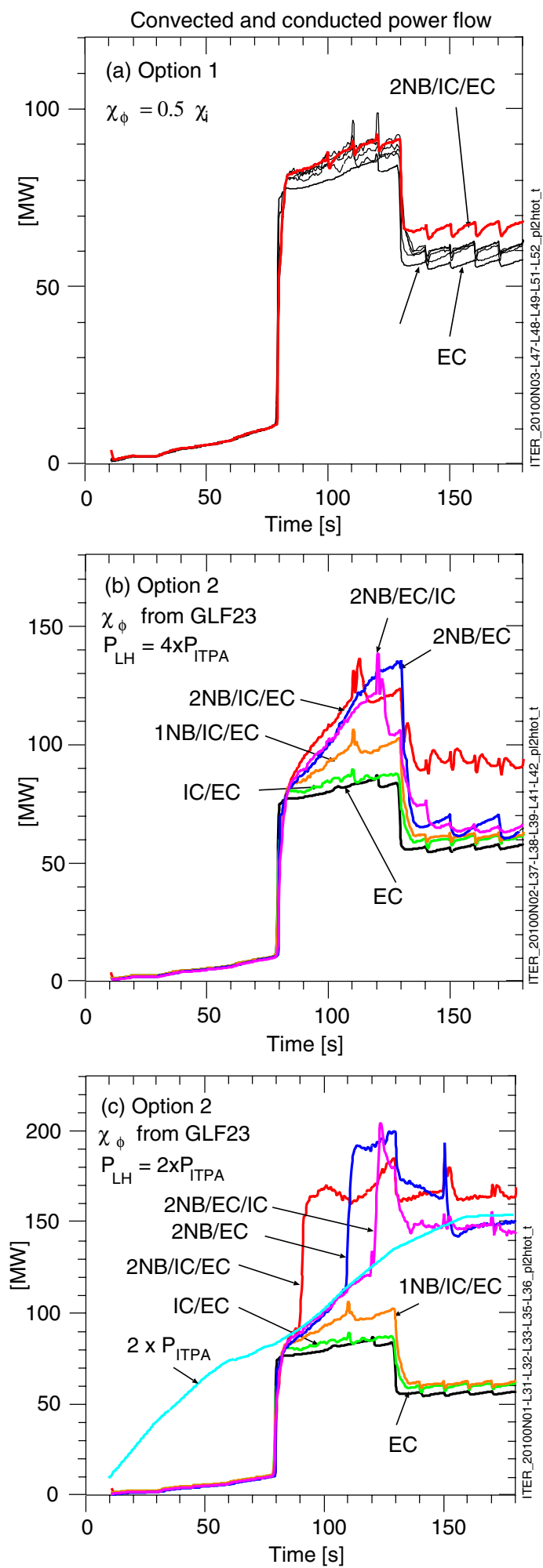

Figure 6. Energy flow near the boundary for the six heating mixes; (a) option 1 with the $\mathrm{L} \rightarrow \mathrm{H}$ power threshold is assumed to be scaled up by a factor of 4 to prevent the H-mode; $(b)$ option 2 with the $\mathrm{L} \rightarrow \mathrm{H}$ power threshold is assumed to be scaled up by a factor of 4 to prevent the H-mode; (c) option 2 with the $\mathrm{L} \rightarrow \mathrm{H}$ power threshold is assumed to be scaled up by a factor of 2 which allows three heating mix cases to achieve H-mode during the density ramp; During the $\mathrm{H}$-mode phase, the pedestal temperature is assumed to increase to a flat top value of $4.4 \mathrm{keV}$, causing the central temperatures and alpha heating to increase as the density increases. Small effects of sawtooth mixing are seen. 

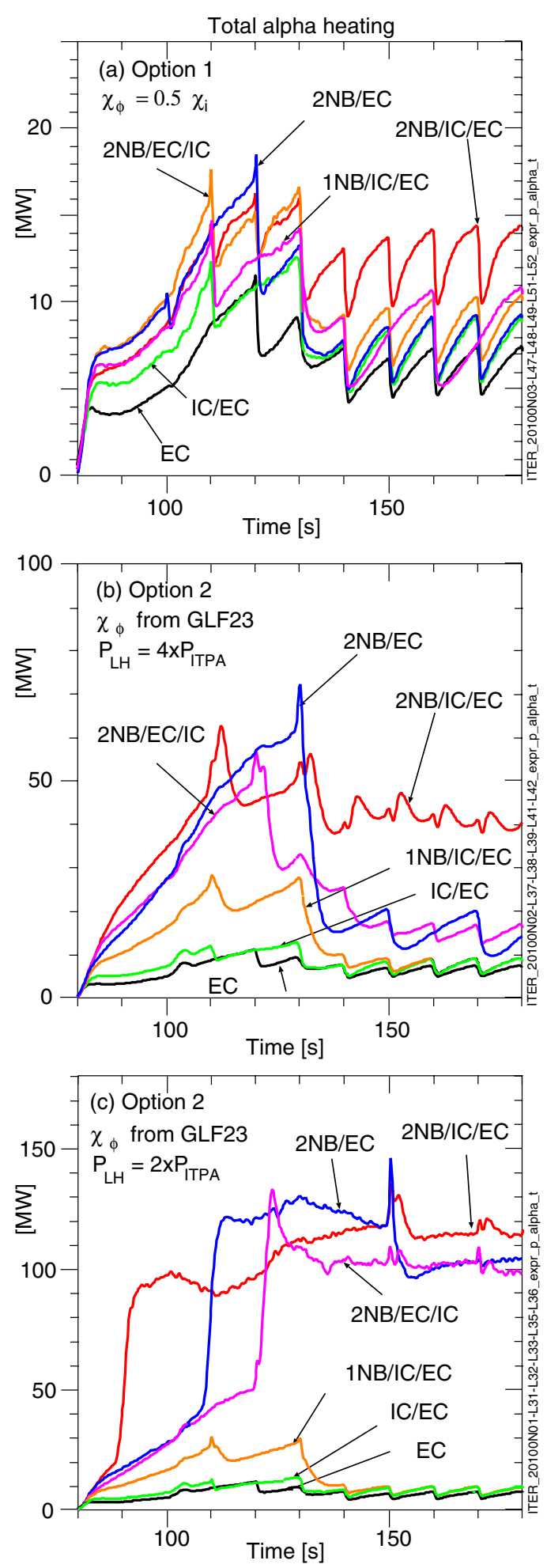

Figure 7. Total alpha power to thermal electrons and ions. (a) option 1 with the $\mathrm{L} \rightarrow \mathrm{H}$ power threshold scaled up by a factor of 4 to prevent the H-mode. Oscillations in $P_{\alpha}$ are caused by sawteeth predicted by Kadomtsev-like mixing at assumed breaks with a $10 \mathrm{~s}$ period. Mixing occurs only if $q$ has just one $q=$ unity surface. ( $b$ ) option 2 with the $\mathrm{L} \rightarrow \mathrm{H}$ power threshold scaled up by a factor of 4 ; (c) option 2 with the $\mathrm{L} \rightarrow \mathrm{H}$ power threshold scaled up by actor of 2. The abrupt increases in the alpha heating in (c) occur at the times of $\mathrm{L} \rightarrow \mathrm{H}$. The total alpha heating power is close to the DT fusion power divided by five except for a dwell in time of approximately $2 \mathrm{~s}$ due to the slowing down of the alpha particles.
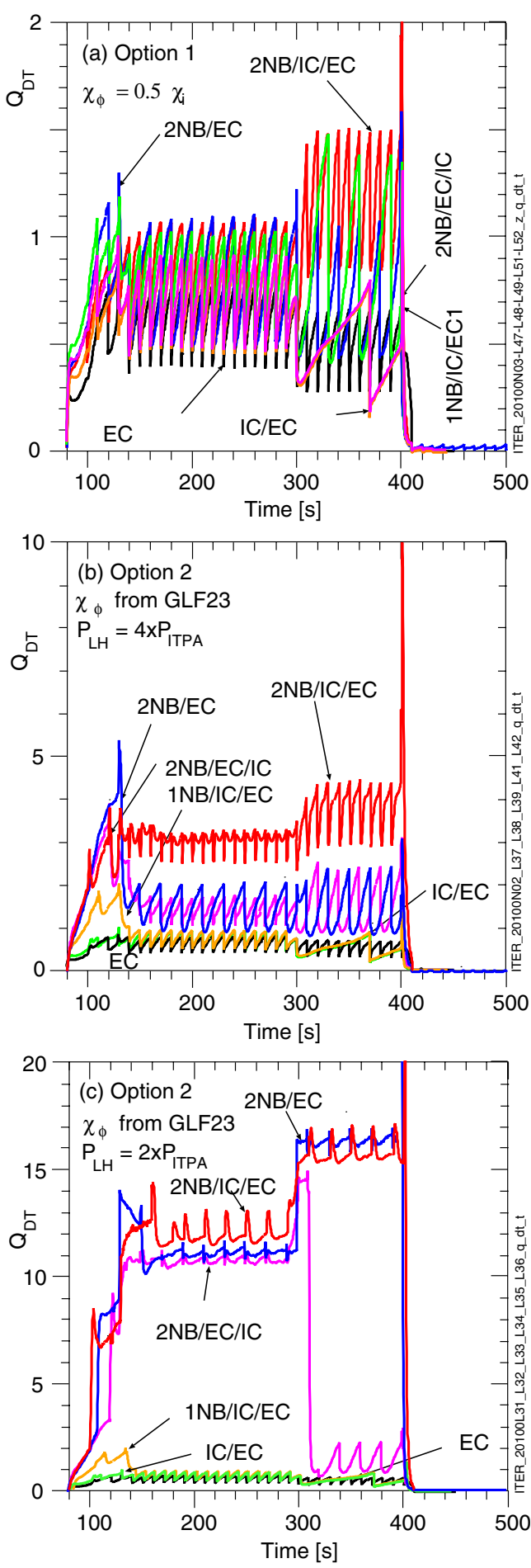

Figure 8. Predictions of $Q_{\mathrm{DT}}$ using $(a)$ option 1; $(b)$ option 2 with $P_{\mathrm{L} \rightarrow \mathrm{H}} / P_{\mathrm{ITPA}}=4 ;(c)$ option 2 with $P_{\mathrm{L} \rightarrow \mathrm{H}} / P_{\text {ITPA }}=2$. In this case three of the mixes remain in the H-mode. Oscillations in $Q_{\mathrm{DT}}$ are caused by sawteeth predicted by Kadomtsev-like mixing at assumed breaks with a $10 \mathrm{~s}$ period. Mixing occurs only if $q$ has just one $q=$ unity surface. The current drive from the NB is predicted to have noticeable effects on the $q$ profile, and thus the mixing radius. The total beam current from $300-400 \mathrm{~s}$ is around $350 \mathrm{kA}$ for the $3 \mathrm{NB} / \mathrm{IC}$ mix, more than twice that of the $2 \mathrm{NB} / \mathrm{EC}$ and $2 \mathrm{NB} / \mathrm{EC} / \mathrm{IC}$ mixes. The predicted $q$ profiles have large radii for $q=$ unity, especially for the $3 \mathrm{NB} / \mathrm{IC}$ mix. The large inversion radii (up to $x$ $\simeq 0.7$ ) cause large oscillations of the central ion temperature and larger regions of high DT fusion reaction rates. 

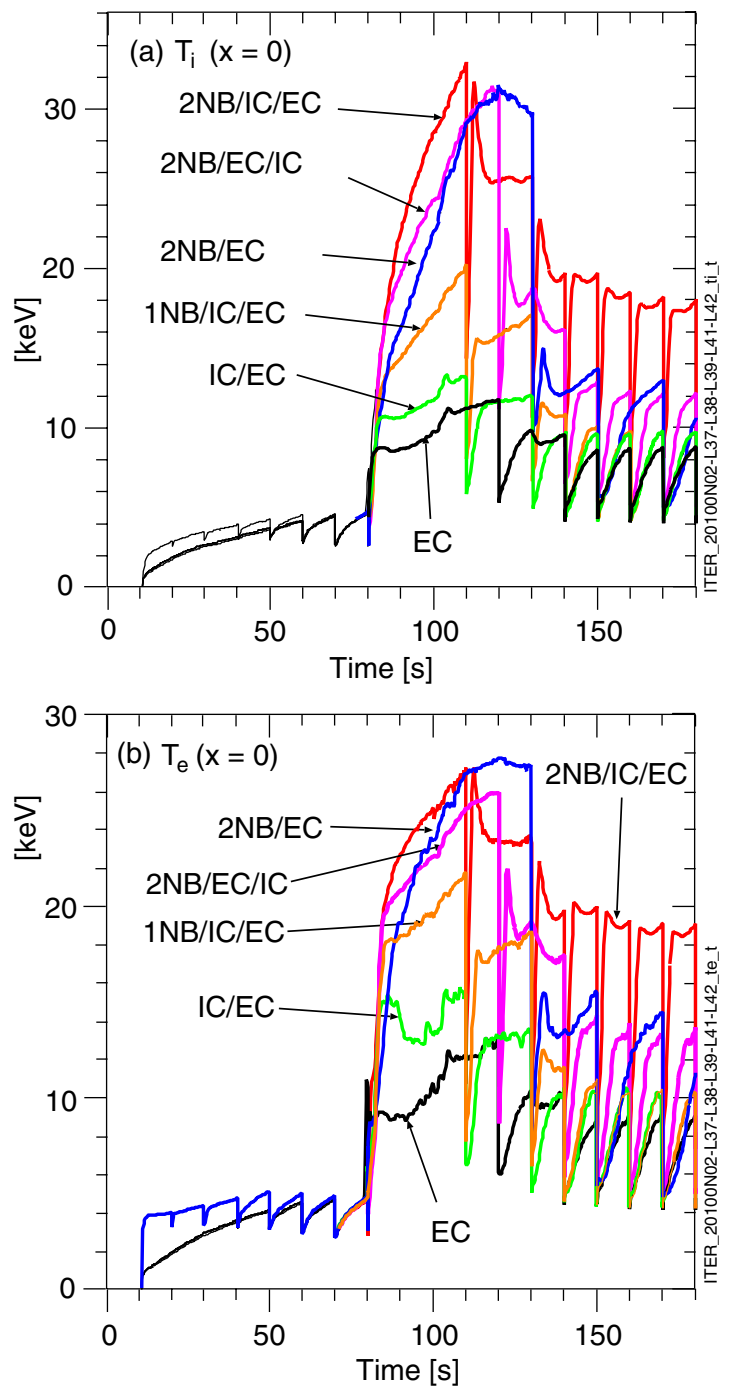

Figure 9. Predictions using option 2 and $P_{\mathrm{L} \rightarrow \mathrm{H}} / P_{\text {ITPA }}=4$ for (a) central ion and $(b)$ electron temperatures.

flow shear suppression. Large central temperatures could be expected in ITER as a consequence of the large minor radius and stiff temperature profiles. If there is a lower bound for the temperature gradients then even with low boundary temperatures, the central ion temperature is predicted to be high. Profiles at one time near peak central temperatures are shown in figure 10 .

The total thermal ion and electron heating powers are plotted in figure $6(b)$. The external heating is relatively constant in the initial phase, but the alpha heating increases as the density and temperatures increase. Plots of $P_{\alpha}$ are shown in figure 7(b). The alpha heating reaches 50-60 MW in heating mixes with two beam lines. The values of $P_{\alpha}$ at the end of the high $P_{\text {ext }}$ phase are listed in table 2.

The alpha heating decreases in the next phase with reduced external power. Profiles of the alpha electron and thermal ion heating for two of the heating mixes are compared in figure 11 with the profiles of the DT power divided by five (the ratio of the kinetic energies of both the alphas and neutrons to the kinetic energies of the alphas when the $\mathrm{He}^{5}$ resonance decays). The $P_{\mathrm{DT}}$ and $P_{\alpha}$ profiles are predicted to have nearly identical shapes except during transients. The $P_{\mathrm{DT}}$ profile
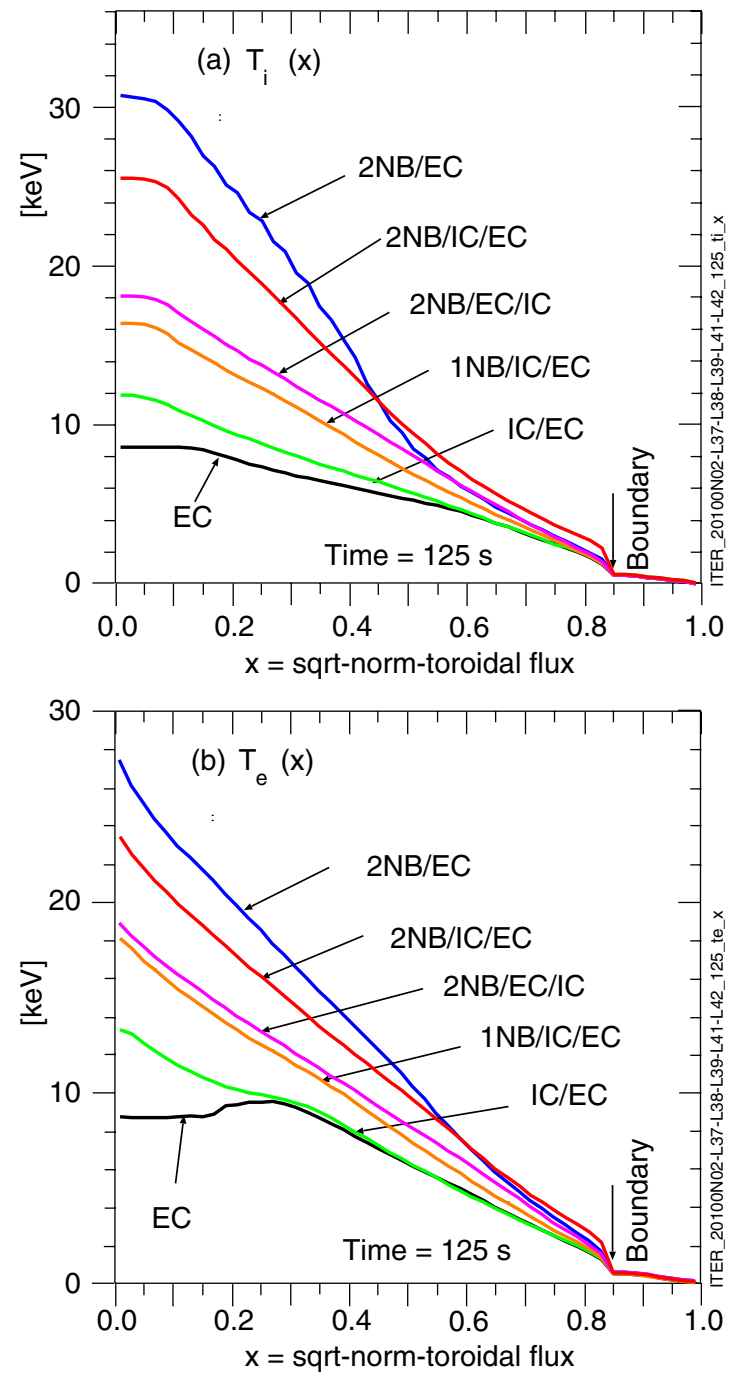

Figure 10. $(a)$ Ion and $(b)$ electron temperature profiles using option 2 and $P_{\mathrm{L} \rightarrow \mathrm{H}} / P_{\mathrm{ITPA}}=4$ near the time when they are largest.

is the instantaneous fusion power whereas $P_{\alpha}$ depends on the slowing down of the alphas. No anomalous diffusion for the fast alpha particles is assumed here, and the classical slowing-down and pitch angle scattering have relatively small effects on their profiles. Results for the heating profiles for the six heating mixes near the peak temperatures are shown in figure 12. The alpha heating profiles are included in the total electron and thermal ion heating profiles. The cases are ordered approximately in diminishing alpha heating and $P_{\mathrm{DT}}$, indicating that central heating is beneficial. Table 2 summarizes $P_{\alpha}$ at the end of the $P_{\text {ext }}=73 \mathrm{MW}$ phase.

The values of $Q_{\mathrm{DT}}$ are shown in figure $8(b)$. The $Q_{\mathrm{DT}}$ is 4.0 for several of the heating mixes with $P_{\text {ext }}=73 \mathrm{MW}$, and 3.5 when $P_{\text {ext }}$ is lowered to $50 \mathrm{MW}$. The times for the three mixes that obtain $\mathrm{H}$-mode are listed in table 2. Note that the mixes with more NB power achieve the H-mode earlier and have higher alpha heating. This shows an advantage of more beam power. In contrast, this advantage is predicted to be more modest during the flat top phases and far from critical values [2].

Next consider option 2 and assume $P_{\mathrm{L} \rightarrow \mathrm{H}}=2 \times P_{\text {ITPA }}$. Three of the heating mixes transition to $\mathrm{H}$-mode and three do 

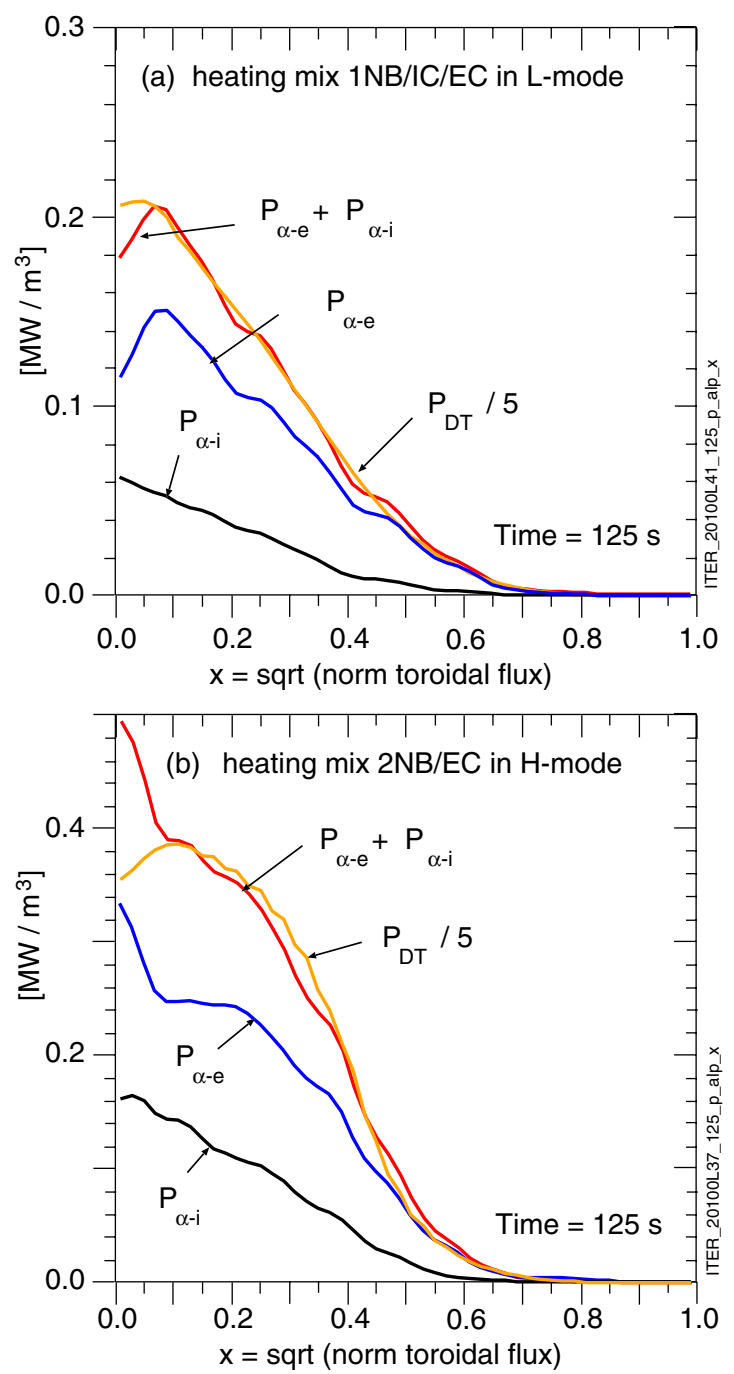

Figure 11. Profiles of alpha powers to thermal electrons and ions and their total compared with the profile of $P_{\mathrm{DT}} / 5$ assuming option 2 when the $\mathrm{L} \rightarrow \mathrm{H}$ power threshold is assumed to be scaled up by a factor of 2. (a) Heating mix that does not achieve H-mode; $(b)$ heating mix that does achieve $\mathrm{H}$-mode. The $P_{\alpha-\mathrm{e}}+P_{\alpha-\mathrm{i}}$ profile is close to the DT fusion power divided by five except for a dwell in time of approximately $2 \mathrm{~s}$ due to the slowing down of the alpha particles. If a non-classical radial shift of fast alphas occurs (due, say to MHD or TAE activity) then the shapes of the $P_{\mathrm{DT}}$ and alpha density profiles would differ.

not. The back transition is assumed to occur if the heat flow decreases below $75 \%$ of $P_{\mathrm{L} \rightarrow \mathrm{H}}$. The mixes achieving $\mathrm{H}$-mode have $P_{\text {heat }}>P_{H \rightarrow L}$ even when $P_{\text {ext }}$ is lowered, so they are predicted to remain in $\mathrm{H}$-mode. The total thermal ion and electron heating powers are plotted in figure $6(c)$, and the total alpha heating in figure $7(c)$. The times of the $\mathrm{L} \rightarrow \mathrm{H}$ are listed in table 2. The total alpha heating is also given in table 2 . Their values increase as the time of transition increases, mainly due to the increase in density. The corresponding results for the total fusion power are shown in figure 7(c). The values of $Q_{\mathrm{DT}}$ are shown in figure $8(c)$ with three of the mixes remaining in H-mode.

Ranges of the volume-averaged normalized pressure $\left\langle\beta_{\mathrm{n}}\right\rangle$, stored energy and confinement parameters are given in table 3 . Here $\left\langle\beta_{\mathrm{n}}\right\rangle$ is the volume-average of $\beta_{\mathrm{n}}$ defined by $\beta_{\text {tor }} a B / I$ where $\mathrm{B}$ is the vacuum magnetic field averaged over the plasma volume, and $\beta_{\text {tor }}$ is defined as $P /\left(8 \pi B^{2}\right)$ with $P$ the local total (thermal and fast ion) pressure. The values for the normalized energy confinement times are compared with values extrapolated from the $H 98 y 2$ scaling relation [33] derived from a multi-tokamak database of ELMing H-mode plasmas maintained by the ITPA confinement working group. The values of $H 98 y 2$ measured in high confinement $\mathrm{H}$-mode plasmas tend to be around 1.0, and for L-mode plasmas around 0.5. The values computed from the PTRANSP runs range from 0.24 to 0.33 with option 2 , and 0.24 to 0.40 with option 1. These are lower than $0.5 \times H 98 y 2$ as a consequence of the pessimistically assumed low boundary temperatures shown in figure 4(a).

\section{Results for the H-mode}

For the $\mathrm{H}$-mode predictions the heating mix $2 \mathrm{NB} / \mathrm{IC} / \mathrm{EC}$ is assumed. A study using a different heating mix is given in [15]. GLF23 is used for the plasma temperatures, but not for $v_{\phi}$. The momentum transport is assumed to be related to the ion energy conductivity by $\chi_{\phi} / \chi_{\mathrm{i}}=0.5$, as in option 1 used for the L-mode study. With the PTRANSP-NUBEAM predictions of the NB torques, $v_{\phi}$ is predicted to be relatively low $\left(\simeq 10-20 \mathrm{krad} \mathrm{s}^{-1}\right)$ and the flow-shearing rate is predicted to have little effect on the GLF23-predicted temperatures. The nominal database scaling $P_{\text {ITPA }}$ (equation (1)) is assumed. Consequently the transition occurs immediately after the external heating is applied, precluding prediction of alpha heating in L-mode plasmas except during the ohmic phase.

After the transition to $\mathrm{H}$-mode the pedestal temperatures are predicted by the PEDESTAL module in PTRANSP. Their values are used as boundary values for the GLF23 predictions. The resulting core temperatures depend sensitively on them. Since there is uncertainty about their values, scans were performed scaling the PEDESTAL values to predict a range of temperatures and alpha heating powers.

Examples of $P_{\alpha-\mathrm{e}}$ profiles for the scans are shown in figure 13. The $P_{\alpha-\mathrm{e}}+P_{\alpha-\mathrm{i}}$ time evolutions for the scans are shown in figure 14. They tend to increase following dips after $100 \mathrm{~s}$ when the plasma shape is adjusted, and after $130 \mathrm{~s}$ when $P_{\text {ext }}$ is reduced. The $Q_{\mathrm{DT}}$ time evolutions for the scans are shown in figure 15.

Various parameters such as $Q_{\mathrm{DT}}$, central temperatures and the volume-averaged $\left\langle\beta_{\mathrm{n}}\right\rangle$ are plotted versus the local normalized pressure $\beta_{\mathrm{n} \text {,ped }}$ in figure 16 . The parameter $\beta_{\mathrm{n} \text {,ped }}$ is defined as the local $\beta_{\mathrm{n}}$ at the top of the pedestal, and is proportional to $T_{\text {ped }}$ since the $n_{\mathrm{e}}$ profile is fixed. Results from two phases of the discharges are plotted: a phase with $P_{\text {ext }}$ $73 \mathrm{MW}$ from the time interval 120 to $130 \mathrm{~s}$, and with $50 \mathrm{MW}$ from 240 to $250 \mathrm{~s}$ between two sawteeth.

The scaling of $Q_{\mathrm{DT}}$ with $P_{\mathrm{ext}}$ is approximately $\left(P_{\mathrm{ext}}\right)^{-1.0}$, close to the result $\left(P_{\text {ext }}\right)^{-0.8}$ in [25]. The scaling $Q_{\mathrm{DT}} \propto$ $\left(P_{\mathrm{ext}}\right)^{-1.0}$ implies that $P_{\mathrm{DT}}$ is independent of $P_{\mathrm{ext}}$ so $P_{\alpha}$ dominates. Other PTRANSP predictions for ITER H-mode plasmas assuming peaked density profiles do not exhibit a simple scaling of $Q_{\mathrm{DT}}$ with $P_{\text {ext }}$. For these, very slow relaxations of profiles lead to slow increases in the central temperatures and thus in $P_{\mathrm{DT}}$ so stationary phases are not seen even with constant $P_{\text {ext }}$. A linear scaling of $Q_{\mathrm{DT}}$ with 

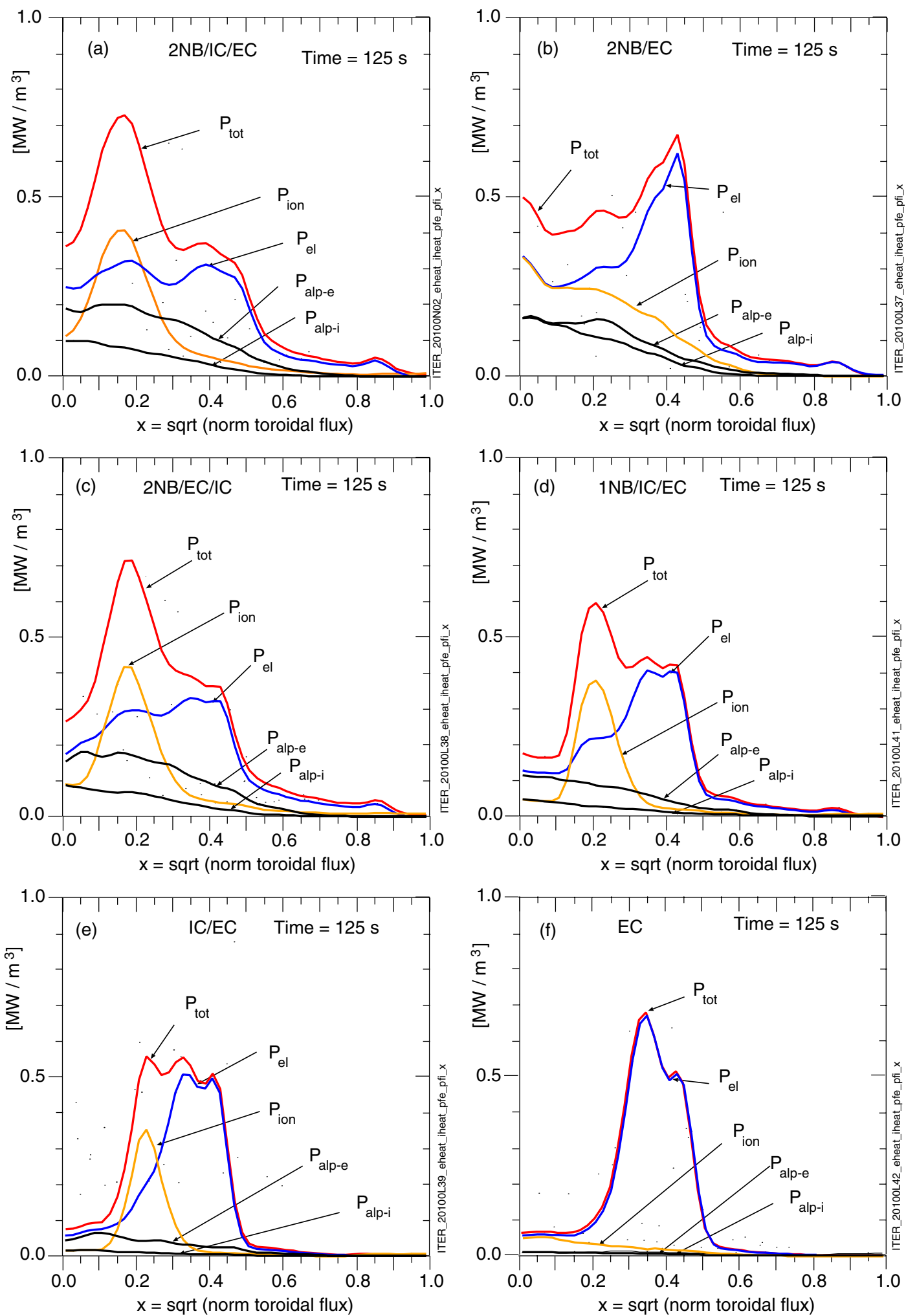

Figure 12. Predictions of electron and ion heating using option 2 late in the L-mode phase, $2 \mathrm{~s}$ before the transition to H-mode. The alpha heating profiles are summed in the total ion and electron heating profiles.

$T_{\text {ped }} \propto \beta_{\mathrm{n} \text {,ped }}$ is found. This linear scaling contrasts the quadratic scaling found in [25]. In that study effects of ash accumulation are ignored, and the heating profile shapes held fixed with only $P_{\text {ext }}$ varied. Dilution from alpha ash accumulation increases with $P_{\mathrm{DT}}$, and thus with $\beta_{\mathrm{n} \text {,ped }}$.
Various effects are expected to limit how large $\beta_{\mathrm{n}, \text { ped }}$ can be. Predictions from modelling of the peeling-ballooning stability of the edge [34] indicates that the value $\beta_{\mathrm{n} \text {,ped }}$ needs to be limited to values below $\simeq 0.6-0.8$. Note that with $P_{\text {ext }}=$ $73 \mathrm{MW}$ figure $16(a)$ predicts $Q_{\mathrm{DT}}<10$ for $\beta_{\mathrm{n} \text {,ped }}<0.8$ 
Table 3. Ranges of values from the six heating mix predictions for the total stored energy and confinement parameters computed for both options are given at two values for $P_{\text {ext }}: 73 \mathrm{MW}$ near $130 \mathrm{~s}$, and $50 \mathrm{MW}$ near $245 \mathrm{~s}$. The ratio of the thermal energy confinement time $\tau_{\mathrm{E}}$ and the expression from the ITPA database, i.e. the $\mathrm{H}$-factor $H 98$ y 2 values are listed.

\begin{tabular}{llllll}
\hline \multirow{2}{*}{$\begin{array}{l}\text { Heating mix } \\
P_{\text {ext }}(\mathrm{MW})\end{array}$} & 73 & 50 & & \multicolumn{2}{c}{ Option 2} \\
\cline { 2 - 3 } \cline { 5 - 6 } \cline { 5 - 6 } & & & 73 & 50 \\
\hline$\left.\beta_{\mathrm{n}}\right\rangle$ & $0.60-0.72$ & $0.55-0.68$ & & $0.60-1.40$ & $0.50-0.90$ \\
$\mathrm{~W}_{\text {tot }}(\mathrm{MJ})$ & $125-138$ & $105-138$ & & $125-300$ & $105-180$ \\
$\tau_{\mathrm{E}}(\mathrm{s})$ & 1.4 & 1.8 & & $1.4-1.6$ & $1.8-2.1$ \\
$H 98 y 2$ & $0.40-0.42$ & $0.38-0.41$ & & $0.38-0.66$ & $0.40-0.53$ \\
\hline
\end{tabular}

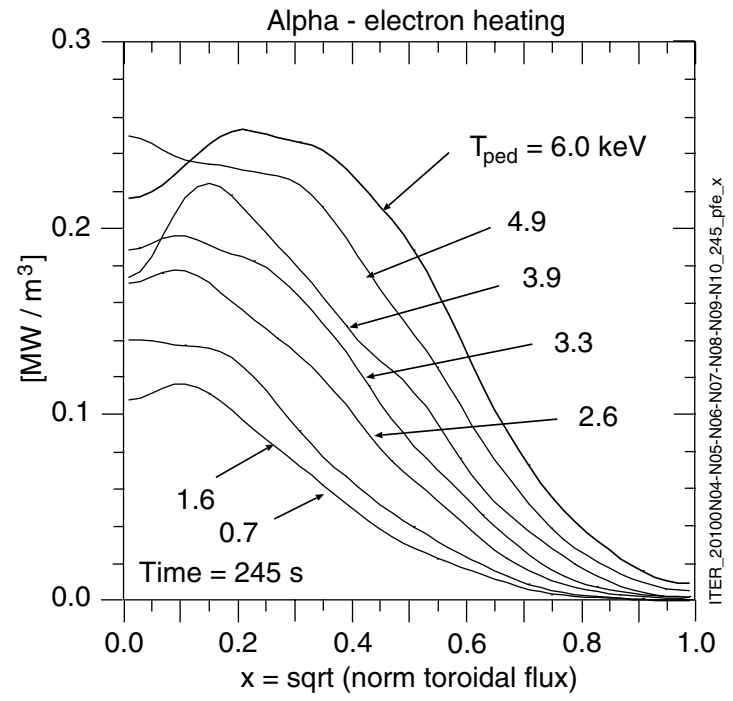

Figure 13. Profiles of $P_{\alpha-\mathrm{e}}$ at the flattop with $P_{\mathrm{ext}}=73 \mathrm{MW}$.

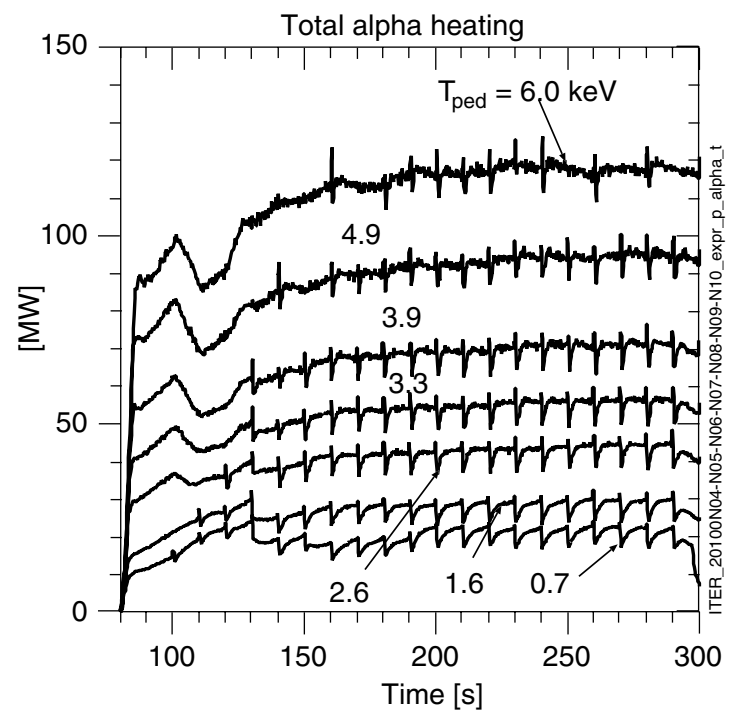

Figure 14. Evolution of the total alpha heating for different assumptions for the pedestal temperature.

(table 4). Values of $Q_{\mathrm{DT}}=10$ appears achievable with $P_{\text {ext }}=50 \mathrm{MW}$ (table 5).

There are other effects that could limit the allowable range of $\beta_{\mathrm{n}, \mathrm{ped}}$. One is NTM activity expected to limit confinement at $\left\langle\beta_{\mathrm{n}}\right\rangle$ above 1.8. Also TAE-driven losses of fast alpha particles

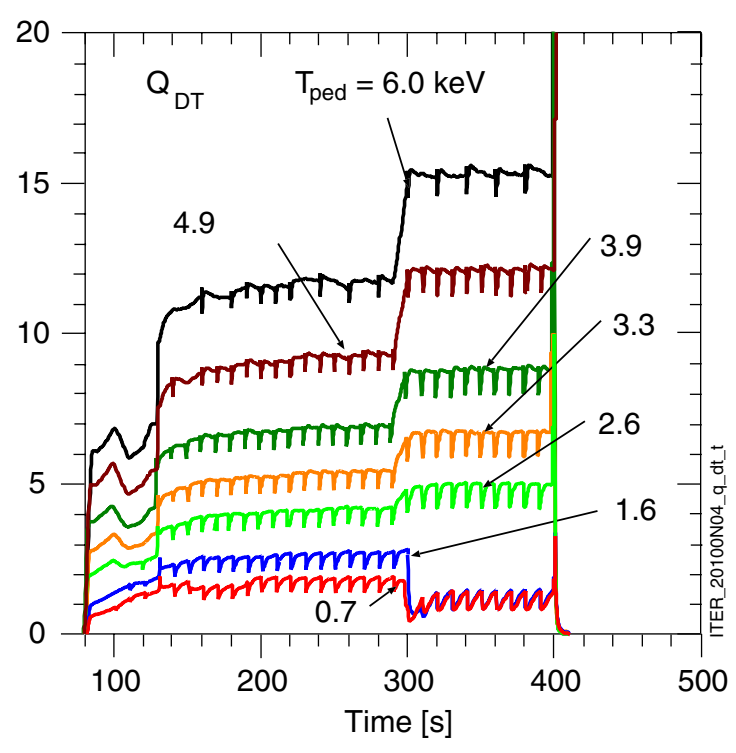

Figure 15. Evolution of $\mathrm{Q}_{\mathrm{DT}}$ for different assumptions for the pedestal temperature.
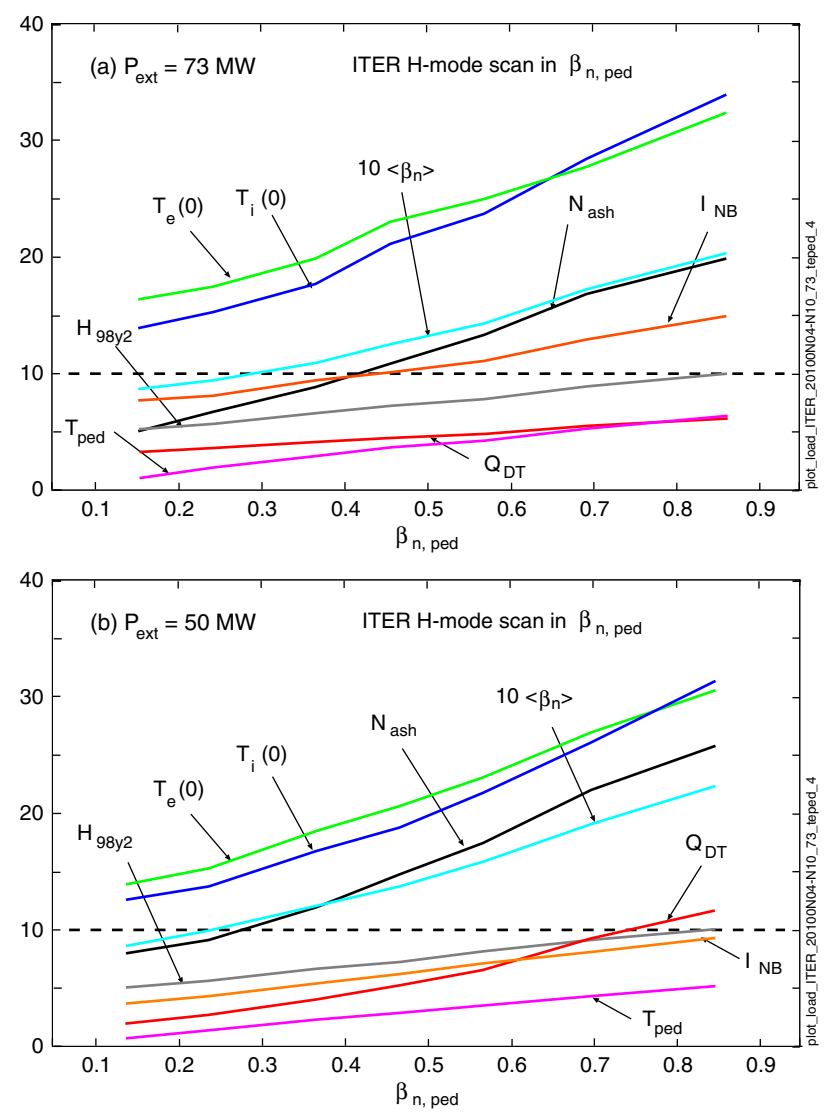

Figure 16. Results with $P_{\text {ext }}$ of (a) $73 \mathrm{MW}(115-120 \mathrm{~s})$; (b) $50 \mathrm{MW}$ $(245 \mathrm{~s})$. The central and pedestal temperatures are in $\mathrm{keV}$, the numbers of ash ions are in $10^{21}$, and the beam-driven currents $\mathrm{I}_{\mathrm{NB}}$ are in 0.1 MA.

are expected if the fast-ion pressure is too large. Another limit to $Q_{\mathrm{DT}}$ is the limit of allowable heat flows to the divertor and vessel. These limiting effects appear near values with $Q_{\text {DT }} \simeq 10$. 
Table 4. Results for the H-mode scan in the early phase with $P_{\text {ext }}=73$ MW. $Q_{\text {DT }}$ is less than 10 since $P_{\text {ext }}$ is large.

\begin{tabular}{llllll}
\hline $\begin{array}{l}T_{\text {ped }} \\
(\mathrm{keV})\end{array}$ & $\begin{array}{l}T_{\mathrm{e}}(0) \\
(\mathrm{keV})\end{array}$ & $\begin{array}{l}T_{\mathrm{i}}(0) \\
(\mathrm{keV})\end{array}$ & $H_{H 98 y 2}$ & $Q_{\mathrm{DT}}$ & $\begin{array}{l}P_{\alpha} \\
(\mathrm{MW})\end{array}$ \\
\hline 6.0 & 35.0 & 33.5 & 0.96 & 6.0 & 70 \\
4.9 & 28.5 & 28.2 & 0.85 & 5.0 & 57 \\
3.9 & 25.1 & 23.5 & 0.74 & 3.7 & 45 \\
3.3 & 23.0 & 21.0 & 0.68 & 2.9 & 36 \\
2.6 & 20.0 & 17.8 & 0.62 & 2.4 & 28 \\
1.6 & 18.0 & 15.9 & 0.53 & 2.3 & 23 \\
0.7 & 17.2 & 14.0 & 0.48 & 2.0 & 20 \\
\hline
\end{tabular}

Table 5. Results for the H-mode scan in the flat top phase with $P_{\text {ext }}=50 \mathrm{MW}$.

\begin{tabular}{llllll}
\hline $\begin{array}{l}T_{\text {ped }} \\
(\mathrm{keV})\end{array}$ & $\begin{array}{l}T_{\mathrm{e}}(0) \\
(\mathrm{keV})\end{array}$ & $\begin{array}{l}T_{\mathrm{i}}(0) \\
(\mathrm{keV})\end{array}$ & $H_{H} 98 y 2$ & $Q_{\mathrm{DT}}$ & $\begin{array}{l}P_{\alpha} \\
(\mathrm{MW})\end{array}$ \\
\hline 5.3 & 30.6 & 31.5 & 1.02 & 11.8 & 87 \\
4.4 & 27.0 & 26.2 & 0.93 & 9.4 & 70 \\
3.6 & 23.2 & 21.9 & 0.83 & 6.7 & 55 \\
3.0 & 20.7 & 18.9 & 0.74 & 5.4 & 43 \\
2.4 & 18.6 & 16.9 & 0.68 & 4.2 & 34 \\
1.5 & 15.4 & 13.9 & 0.58 & 2.9 & 24 \\
0.8 & 14.0 & 12.7 & 0.52 & 2.1 & 19 \\
\hline
\end{tabular}

An identical scan was performed with the NB injection energy reduced from $1 \mathrm{MeV}$ to $0.5 \mathrm{MeV}$, keeping the maximum beam power $33 \mathrm{MW}$. The present design for the NB system could allow reduction of the beam voltage by re-gaping the accelerator grids, but the total current cannot be increased by much so the total NB power would be about $16 \mathrm{MW}$. The NB heating, torquing, and current drive profiles shift outwards. The predicted core temperatures, alpha heating and $Q_{\mathrm{DT}}$ are slightly higher due to increased flow shearing. The current drive is reduced by a factor of two below the values shown in figure 16 .

\section{Summary}

ITER L-mode plasma performance is studied assuming six heating mixes and a range of physics assumptions. The power threshold for the $\mathrm{L} \rightarrow \mathrm{H}$ transition is assumed to be higher than the maximum external heating power planed ( $73 \mathrm{MW})$. The GLF23 model is used to predict temperatures. Two alternative options are used to compute the flow-shearing rate to give a range of possibly results in ITER. Option 1 uses the conservative assumption that $\chi_{\phi} / \chi_{i}=0.5$. Option 2 uses GLF23 to predict $v_{\phi}$, which predicts considerable higher $v_{\phi}$ rates and higher $P_{\mathrm{DT}}$ as a result of the predicted flow-shearing suppression of transport. Examples are given of conditions with $\mathrm{L} \rightarrow \mathrm{H}$ transitions when $P_{\mathrm{ext}}+P_{\alpha}$ exceeds the threshold. Values of $P_{\alpha}=12-65 \mathrm{MW}$ in L-mode are predicted.

These results suggest that having as much NB power as possible is a good strategy for ITER. If the $\mathrm{L} \rightarrow \mathrm{H}$ power threshold is high, and if the flow-shearing rate is high, as with the optimistic option, then extra NB power could be crucial for the H-mode.

Predictions for the H-mode use the conservative option 1. The alpha heating as well as the total fusion power and $Q_{\mathrm{DT}}$ are predicted to depend sensitively on the pedestal temperature. The $P_{\mathrm{DT}}$ increases approximately linearly with the pedestal temperature. For pedestal pressures compatible with peelingballooning simulations, $P_{\alpha}$ up to $80 \mathrm{MW}$ are predicted.

Improvements in theory and modelling that would strengthen the validity of the predictions are (1) better models are needed for predicting plasma temperatures, $v_{\phi}$, and density. Density profiles are coupled with performance, so predictions of the transport, including pinches and nondiagonal terms such as thermo-electric diffusivities of the various plasma species are needed. Intrinsic rotation and neoclassical viscosity might be important in ITER. Having the TGLF model coupled to a time-dependent integrated predictive model would be an important improvement over the GLF23 model used here. (2) Better models of anomalous fast-ion transport are needed. These should include effects of MHD, NTM and TAE modes. (3) Improved predictions of the heating, torquing and current drive are needed for source terms in the transport calculations.

\section{Acknowledgments}

This paper is dedicated to Glenn Bateman who died on 5 August 2011 after a long struggle with cancer. Glenn was passionately dedicated to improving predictive integrated modelling of tokamak plasmas. He was one of the first to realize consequences of stiff profiles. Some of his research led to important improvements to PTRANSP. His death is a loss to magnetic confinement energy research.

The author wishes to thank P. Thomas, F. Wagner and S. Zweben for motivating this work, and the PTRANSP development teams at PPPL, Lehigh, GA and LNL.

This work is supported by the US Department of Energy contract DE-AC02-09CH11466.

\section{References}

[1] Aymar R., Chuyanov V., Huguet M., Shimomura Y., the ITER Joint Central Team and Home Teams 2001 Nucl. Fusion 411301

[2] Budny R.V., Andre R., Bateman G., Halpern F.D., Kessel C., Kritz A. and McCune D. 2008 Nucl. Fusion 48075005

[3] Halpern F.D., Kritz A.H., Bateman G., Pankin A.Y. and Budny R.V. and McCune D.C. 2008 Phys. Plasmas 15062505

[4] Budny R.V. 2009 Nucl. Fusion 49085008

[5] Kramer G. and Budny R.V. 2006 Nucl. Fusion 46 S846

[6] Wang G. et al 2010 Rev. Sci. Instrum. 8110 D908

[7] Diem S.J. et al 2010 Rev. Sci. Instrum. 81 10D914

[8] Gorelenkov N.N., Berk H.L. and Budny R.V. 2005 Nucl. Fusion 45226

[9] Martin Y.R. and ITPA CMDB H-mode Threshold Database Working Group 2008 J. Phys. Conf. Ser. 123012033

[10] Hubbard A. et al 1998 Plasma Phys. Control. Fusion 40689

[11] Ryter F. et al 2009 Nucl. Fusion 49062003

[12] Gohil P., Evans T.E., Fenstermacker M.E., Ferron J.R., Osborne T.H., Park J.M., Schmitz O., Scoville J.T. and Unterberg E.A. 2010 Nucl. Fusion 51103020

[13] Maggi C.F. et al 2011 L-H threshold at low density and low momentum input in the JET tokamak Proc. 38th EPS Conf. (Strasbourg, France, 2011) and http://ocs.ciemat.es/EPS2011PAP/html/

[14] Onjun T., Bateman G., Kritz A.H. and Hammett G. 2003 Plasma Phys. Control. Fusion 451939

[15] Budny R.V. 2011 Predictions of alpha heating in ITER L-mode and H-mode plasmas Proc. 38th EPS Conf. (Strasbourg, France, 2011) and http://ocs.ciemat.es/EPS2011PAP/html/ 
[16] Wagner F. et al 2010 Plasma Phys. Control. Fusion 52124044

[17] Pankin A., Bateman G., Budny R., Kritz A., McCune D., Polevoi A. and Voitsekhovitch I. 1981 Comput. Phys. Commun. 4361

[18] Brambilla M. 2002 Plasma Phys. Control. Fusion 442423

[19] Budny R.V. et al 2010 Proc. IAEA Conf. (Daejeon, Korea, 2010)

[20] Batchelor D.B. and Goldfinger R.C. 1980 Nucl. Fusion 20403

[21] Kritz A.H., Hsuan H., Goldfinger R.C. and Batchelor D.B. 1982 Conf. Proc. 3rd Int. Symp. on Heating in Toroidal Plasmas ECE (Brussels, Belgium) vol 2, p 707

[22] Prater R., Farina D., Gribov Y., Harvey R.W., Ram A.K., Lin-Liu Y.-R, Poli E., Smirnov A.P., Volpe F., Westerhof E., Zvonkov A. and the ITPA Steady State Operation Topical Group 2008 Nucl. Fusion 48035006

[23] Budny R.V. et al 2000 Phys. Plasmas 75038

[24] Waltz R.E., Staebler G.M., Dorland W., Hammett G.W., Kotschenreuther M. and Koning J.A. 1997 Phys. Plasmas 42482
[25] Kinsey J.E., Staebler G.M., Waltz R., Candy J. and Budny R.V. 2011 Nucl. Fusion 51083001

[26] Kinsey J.E., Staebler G.M. and Waltz R.E. 2002 Phys. Plasmas 91676

[27] Staebler G. and St. John H. E. 2006 Nucl. Fusion 46 L6

[28] Staebler G., Waltz R.E. and Kinsey J.E. 2011 Phys. Plasmas 18056106

[29] Houlberg W.A., Shang K.C., Hirshman S.P. and Zarnstorff M.C. 1997 Phys. Plasmas 43230

[30] Anderson H. et al 2000 Plasma Phys. Control. Fusion 42781

[31] Summers H. P. 'The ADAS User Manual v2.6', http://www.adas.ac.uk/manual.php

[32] Stratton B.C., Fonck R.J., Stratton G.R., Budny R.V., Chang Z., Wising F. and Ödblom A. 1996 Nucl. Fusion 361586

[33] ITER Physics Basis Editors et al 1999 Nucl. Fusion 392137

[34] Snyder P.B., Wilson H.R., Osborne T.H. and Leonard A.W. 2004 Plasma Phys. Control. Fusion 46 A131 